\title{
Experiments Concerning Nonequilibrium Conductivity in a Seeded Plasma
}

\author{
E. E. Zukoski, ${ }^{*}$ T. A. Cool, $\dagger$ And E. G. Gibson $\dagger$ \\ Caiifornia Institute of Technology, Pasadena, Calif.
}

\begin{abstract}
Measurements of conductivity have been made in a plasma composed of argon seeded with potassium vapor. The gas temperature was $2000^{\circ} \mathrm{K}$; the pressure, $1 \mathrm{~atm}$; and the potassium concentration was between 0.22 and 0.80 mole-percent. Conductivity values, calculated from a two-temperature model in which the energy dependence of the cross sections and radiation losses from the plasma are taken into account, agree well with experimental values. Measured values of the plasma temperature appear to be about $10 \%$ less than predicted values. Relaxation times for the conductivity in response to a step function change in the electric field were proportional to $\left(n_{\mathrm{e} 0} / \sigma_{0} E^{2}\right)$ and were a few tens of microseconds for a field strength in the range 3 to $10 \mathrm{v} / \mathrm{cm}$. The ionization rate appeared to be limited primarily by the heating rate for the plasma, and the short relaxation times suggest that ionization occurs by a multistep process. Analysis of conductivity and light intensity data obtained during the transient period indicates that the electron temperature approaches its final value during the first few microseconds.
\end{abstract}

\section{Nomenclature}

$c \quad=$ electron speed

$\boldsymbol{c}=$ mean electron speed

$E=$ electric field strength, $\mathrm{v} / \mathrm{cm}$

$e=$ electron charge

$f_{0}=$ electron velocity distribution function

= Planck's constant

$=$ current density, $\operatorname{amp} / \mathrm{cm}^{2}$

$=$ Boltzmann constant

$=$ atomic or electronic mass

$=$ number density

$Q_{i}=$ diffusion cross section of $i$ th species, see Eqs. (3) and (8) for averaged quantities

$T=$ temperature

$V_{i}=$ ionization potential

$\epsilon=$ mean thermal energy, $\frac{3}{2} k T$

$\bar{\nu}_{i}=$ averaged collision frequency, see Eq. (8)

$\sigma=$ electrical conductivity

$\sigma_{c}=$ close-encounter conductivity term

$\sigma_{s}=$ Spitzer conductivity term

$\Sigma_{m}=$ sum over both neutral and ionized species

$\Sigma_{n}=$ sum over neutral species

$\tau_{c}=$ characteristic time $\left(n_{e 0} V_{i} / \sigma_{0} E^{2}\right)$

$\tau_{r}=$ relaxation time for conductivity

$\begin{aligned} & \tau_{r} \\ & \dot{\Omega}\end{aligned}=$ rate of elastic energy loss per unit volume from free electrons

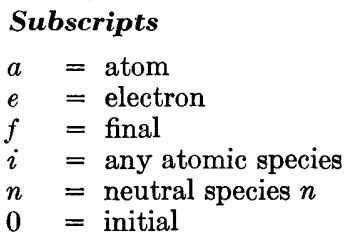

\section{Introduction}

$\mathbf{I}^{\mathrm{N}}$ recent years, interest has been shown in the use of electrically conducting fluids as working media for propulsion de vices or power generating systems. In both devices, elec-

Presented as Preprint 64-28 at the AIAA Aerospace Sciences Meeting, New York, January 20-22, 1964; revision received May 20,1964 . This work was supported by the Air Force Office of Scientific Research under Grant No. AF-AFOSR-160-63.

* Associate Professor of Jet Propulsion, Daniel and Florence Guggenheim Jet Propulsion Center. Associate Fellow Member AIAA.

† Graduate Student in Mechanical Engineering, Daniel and Florence Guggenheim Jet Propulsion Center. Student Member AIAA. tric fields of appreciable strength are required to drive a current in the gas. The presence of such fields tends to elevate the electron temperature and to lead to a degree of ionization which is greater than that corresponding to the original gas temperature. This enhanced ionization can lead to appreciable increases in electric conductivity of the gases and hence to more efficient propulsion and generating devices. These nonequilibrium effects have been studied in conjunction with generator and accelerator problems by a number of authors ${ }^{1-3}$ when the plasma is a noble gas seeded with an alkali metal vapor.

The experiments discussed in this paper deal with measurement of the electrical conductivity and the response of the conductivity to a step function change in applied electric field for a system in which a relatively cool, seeded plasma is used as the working medium.

The measurements were made in a plasma flowing through a circular duct with electrodes arranged to give an axial current. The plasma was composed of argon gas seeded with potassium vapor, and measurements were made at a pressure of $1 \mathrm{~atm}$ and a gas temperature of about $2000^{\circ} \mathrm{K}$. A voltage pulse technique was used, and the relaxation phenomena occurred during periods that were short compared with the residence times of the gas between the electrodes. The joule heating of the neutral gas during the transient periods investigated was small.

Conductivity values were calculated from measurements of voltage and total current made during the steady-state period after the transient had died out. Changes in emission of two spectral regions were measured, and the steady-state values were used to estimate the electron temperature change in response to the voltage pulse. Conductivity and intensity data obtained during the pulse were used to obtain a crude picture of the phenomena occurring in the plasma during the transient period.

\section{Apparatus}

An arcjet heater is used to heat the larger portion of an argon gas flow. This main flow is then combined with a smaller, secondary flow of argon which has been passed through a potassium boiler, with a minimum residence time of $2 \mathrm{sec}$, and is saturated with potassium vapor. The flow passes through a mixing chamber with a length-to-diameter ratio of 16 before entering the test section.

The concentration of potassium in the flow, which results from the combination of these two streams, is fixed by con- 
trolling the boiler temperature and secondary argon flow rate. Measurements indicate that the potassium concentration in the resulting stream is within $10 \%$ of the calculated flow rates. A much more detailed description of the equipment for the general flow system is given by Pinchak. ${ }^{4}, 5$

During the tests, the plasma pressure was maintained at $1 \mathrm{~atm}$ and the argon flow rate at $2.4 \mathrm{~g} / \mathrm{sec}$. For the test section conditions used, this mass flow corresponds to a speed of about $300 \mathrm{fps}$, or a Mach number of about 0.10 . The plasma temperature was $2000 \pm 100^{\circ} \mathrm{K}$, and the potassium seed concentration was kept in the range between 0.2 and 0.8 mole $\%$. Measurements made at the end of the test section showed that the temperature profile there was flat and fell off 5 to $10 \%$ as the wall was approached.

A typical test section is shown in Fig. 1. Electrons are emitted thermionically from a spiral tungsten electrode immersed in the flow at the downstream end of the cylindric test chamber and flow axially to the anode that is a stainlesssteel cylinder imbedded in the insulating wall. The test section inside diameter was $\frac{1}{2}$ in.; typical dimensions of the other elemener are shown in Fig. 1. Tungsten-wire voltage probes of 0.060 -in. diam were inserted as shown. The voltage drop between these electrodes rather than that between the cathode and anode was used to reduce end-effect errors due to electrode geometry.

The insulating cylinder used as the test-section wall was made of boron nitride or alundum, and it was thermally shielded so that the inner wall temperature was above $1200^{\circ}$ K. The electric circuit, shown schematically in Fig. 2, was arranged to allow the application of a step function increase in voltage between the electrodes. A capacitor was used as the voltage source, and the voltage rise time at the electrodes was less than $2 \mu \mathrm{sec}$. The voltage could be maintained at a fixed level for periods up to 1 msec. This system was slightly underdamped, and the voltage traces showed a slight overshoot that was never more than $5 \%$. The applied field strength could be adjusted between 0 and $12 \mathrm{v} / \mathrm{cm}$, and the maximum current density was about $80 \mathrm{amp} / \mathrm{cm}^{2}$. Voltage and current measurements were made with an oscilloscope, and the response time of this equipment was about $1 \mu \mathrm{sec}$.

Measurements were made during a few of the pulses of the intensity changes of a number of spectral lines. The observed radiation was obtained by looking along a diameter of the insulating tube at a point midway between the electrodes. Type 7102 photomultiplier tubes were used to monitor spectral regions centered at $7680 \AA$ and $6900 \AA$, and the half-width of the region observed was fixed by filters at about $90 \AA$. Care was taken to eliminate interaction between the tubes and stray fields produced by the voltage pulsing apparatus. The response time of the tubes and associated circuitry was found experimentally to be less than $2 \mu$ sec.

The pulse was applied when the current and field between the electrodes was zero. The total current, the voltages developed between the electrodes and the two probes, and the intensity of several spectral lines were all obtained as a function of time. Measurements were also made of the steadystate operating conditions of the arc heater and potassium boiler so that the conditions of the gas in the discharge region prior to application of the voltage pulse could be determined.

The electrical conductivity was calculated from the measured values of the total current and values of the electric field strength between the probes. Current density values were obtained by using the actual cross-sectional area of the test section and the assumption that the current density was uniform across the flow. It should be noted that the effective cross-sectional area may be somewhat less than the physical cross-sectional area used here because of the possible existence of current nonuniformities and the neglect of the thermal boundary layer at the duct wall,

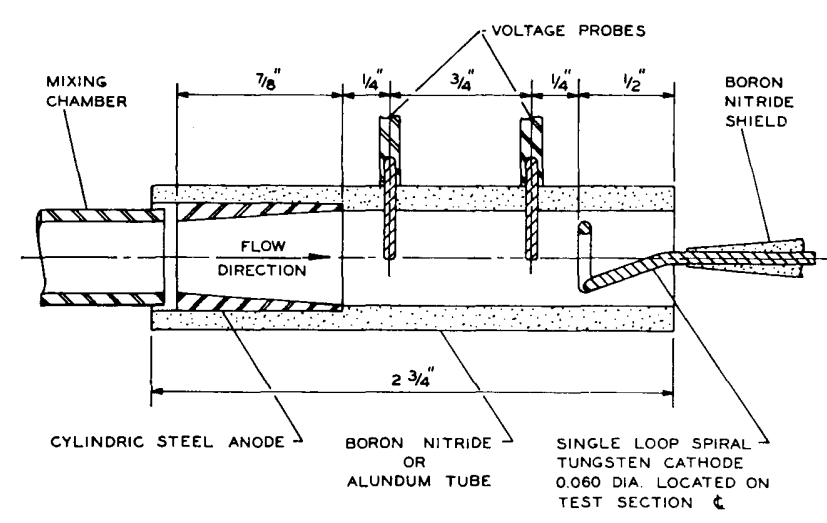

Fig. 1 Schematic diagram of test section.

The length of time required for the discharge to reach a steady state was less than $60 \mu$ sec for most of the experiments. The residence time of gas flowing between the electrodes was greater than $400 \mu$ sec. Hence, for most of the experiments, the gas could be treated as a stationary mass, and phenomena associated with the transport of the plasma could be ignored. In addition, the rate of power input to the plasma was so small that the heating of the neutral gas could be ignored.

\section{Electrical Conductivity}

The method used to calculate values of the scalar electrical conductivity for comparison with the experimental results is discussed in this section. Theoretical description of the transport processes occurring in a slightly ionized gas is complex, and only a brief outline can be given here. Calculations of electrical conductivity in an ionized gas reduce to calculations of effects resulting from perturbations of the electronic velocity-distribution function under the influence of externally-applied electric fields.

Two expansion techniques have been used to determine the electrical conductivity in terms of the electron distribution function and the effects of binary collisions. The first, based on the Enskog procedure, was applied by Chapman and Cowling. ${ }^{6} \quad$ The second, a somewhat more rapidly converging procedure based on the expansion of the distribution function in spherical harmonics, was discussed by Allis. ${ }^{7}$ The latter technique is preferred here, since an arbitrary energy dependence of the cross section for collisions can be easily included in the analysis. This is particularly important for the potassium-argon system because of the pronounced dependence of the cross sections on quantum-mechanical effects.

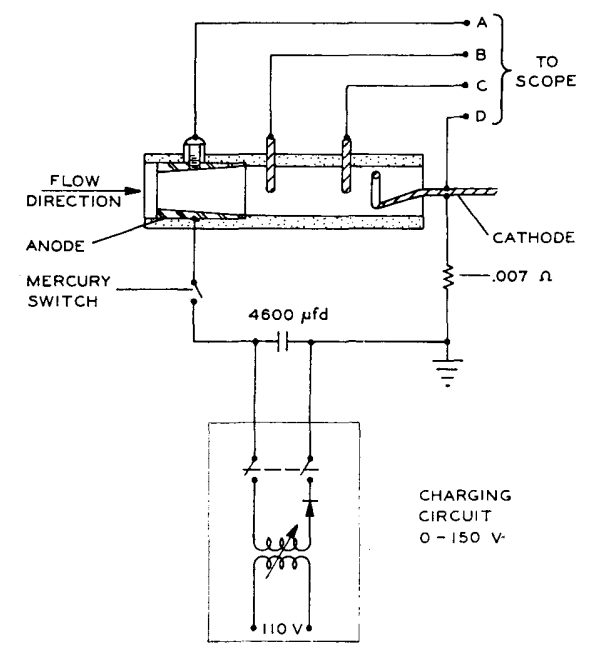

Fig. 2 Schematic diagram of electric circuit. 
The desired scalar conductivity expression is obtained with minor manipulation from Eq. (31.9) of Allis. ${ }^{7}$ The result for elastic collisions between electrons and neutral atoms is

$$
\sigma_{c}=\left(-\frac{4 \pi}{3}\right) \frac{e^{2}}{m_{e}} \int_{0}^{\infty} \frac{d f_{0}}{d c}\left[\frac{c^{2}}{\Sigma_{n} n_{n} Q_{n}}\right] d c
$$

It has been assumed in deriving this result that the heavy species of the plasma have an isotropic distribution and that the mass of the electron is negligible compared to that of the heavy particles. Here $f_{0}$ is the first approximation to the electronic distribution function which is not necessarily the Maxwell distribution function. However, as explained in the next section, the conditions for the attainment of a Maxwellian distribution are probably satisfied for the present experiments, and, for this case,

$$
\sigma_{c}=\frac{(8 / \pi)^{1 / 2} e^{2} n_{e}}{3\left(k T_{e} m_{e}\right)^{1 / 2}} \frac{1}{\overline{\Sigma_{n} n_{n} Q_{n}}}=\frac{0.532 e^{2} n_{e}}{\left(k T_{e} m_{e}\right)^{1 / 2} \overline{\Sigma_{n} n_{n} Q_{n}}}
$$

when the definition

$$
\frac{1}{\overline{\Sigma_{n} n_{n} Q_{n}}} \equiv \int_{0}^{\infty} \frac{\xi e^{-\xi} d \xi}{\Sigma_{n} n_{n} Q_{n}(\xi)}
$$

is used, and $\xi=\frac{1}{2} m_{e} c^{2} / k T$. Note that the average values of the reciprocal sum of the diffusion cross sections appearing here are not the simple averages over the velocity distribution used by several other authors. ${ }^{8}$ From the point of view of the present analysis, the latter average is clearly incorrect, and, for gases such as potassium or argon, the averaged cross-sectional values change appreciably with the average used.

It is interesting to note that the result of evaluating Eq. (2) for cross sections independent of electron speed gives an equation for the conductivity which is identical to that obtained by Chapman and Cowling by use of three terms in the Chapman-Enskog expansion.

If the Coulomb cross section is used in Eq. (2) instead of the electron-neutral cross section, a first approximation to the conductivity of an ionized gas is obtained. Spitzer ${ }^{9}$ has shown that, because of the effect of electron-electron interactions on the distribution function, this value is reduced by a factor of $\gamma_{E}=0.582$ and the resulting conductivity is

$$
\sigma_{s}=\frac{8(2)^{1 / 2}\left(k T_{e}\right)^{3 / 2} \gamma_{\underline{q}}}{\pi^{3 / 2} m_{e}^{1 / 2} e^{2} \ln \Lambda^{2}} \text { where } \Lambda^{2}=\frac{9\left(k T_{e}\right)^{3}}{8 \pi n_{e} e^{6}}
$$

A fundamental difficulty arises in the calculation of conductivity in a plasma for which both close encounters and distant encounters simultaneously interact to determine the resultant conductivity. This is because the first approximation to the electron-distribution function used in calculating $\sigma_{c}$ was the Maxwell distribution whereas that used to obtain Eq. (4) is not. The difference in values of $\sigma_{s}$ calculated by use of the Maxwellian distribution and the modified distribution function used by Spitzer is just $\gamma_{E}=0.582$. Clearly, the difference in distribution function is important here, and, therefore, the deviation from the Maxwellian form may have an appreciable effect on the calculated conductivity when $\sigma_{s}$ and $\sigma_{c}$ are of a similar magnitude. Although several attempts have been made to treat this calculation, e.g., Ref. 10, no calculation is known to the authors which accounts for the change in the distribution function.

To obtain an approximation for the conductivity in this region, the conventional assumption is made that the resultant conductivity is

$$
1 / \sigma=1 / \sigma_{c}+1 / \sigma_{s}
$$

The electron density $n_{e}$ appears as a factor in the conductivity expressions. When the degree of ionization is in equilibrium with the electron temperature, $n_{e}$ can be evaluated from the Saha equation. In the present experiments, only potassium is ionized, and the Saha equation can be written, for the temperatures encountered here, as

$$
n_{e}{ }^{2}=\left(\frac{2 \pi m_{e} k T}{h^{2}}\right)^{3 / 2}\left(n_{K 0}-n_{e}\right) e^{-4.34 / K T}
$$

When the plasma composition, electron temperature, and cross sections are specified, Eqs. (2-6) can be used to calculate the electron density and conductivity.

\section{Two-Temperature Case}

Under certain conditions, the assumption that all the species in the plasma are in thermal equilibrium breaks down. This can occur because energy is supplied to the plasma primarily by the action of the electric field on the electron gas, and it must be distributed to other species by interaction with the electrons. The population of the excited states may be in thermal equilibrium with the electron gas when the electron density is high enough. However, the transfer of energy between electrons and the thermal component of atomic energy is relatively very inefficient (because of the great difference in masses of the electron and atomic species), and, hence, the atom translational temperature may be much less than the electron temperature.

The situation is actually more complex, since radiant energy losses from the various excited states may be so important that the population of the excited states would be out of thermal equilibrium with either electron gas or atomic species, and the "temperature" of any excited level could vary.

In the present approach, this complex picture is radically simplified by assuming that the electrons and excited atomic states are in thermal equilibrium at a temperature that can be greatly different from the translational temperature of the atomic species. In addition, it is assumed that the distribution functions for electron and atomic velocities are Maxwellian to the first approximation. The concept of a twotemperature plasma was applied to electron diffusion through gases some time ago, e.g., Ref. 11. However, the application of this model to the present problem was brought to the authors' attention by the work of Kerrebrock, ${ }^{1}$ who added the key suggestion that the electron gas could be considered to be in thermal equilibrium with the population of the excited states. A great many assumptions are implicit in this two-temperature model of the plasma, and it is beyond the scope of this paper to justify all of them. A few of the more important assumptions are discussed here.

First, in order that the electron gas maintain an approximate Maxwellian distribution, it is necessary that 1) the energy gained between collisions from the electric field be small compared with the mean thermal energy and 2) that the electron-electron collision frequency be high enough to insure that the energy exchange between electrons dominates energy loss processes. For the experimental conditions, both 1 and 2 are satisfied.

Second, the populations of the free and bound electronic states are assumed to be determined by the condition of thermal equilibrium at the electron temperature. Establishment of the validity of this assumption requires a detailed knowledge of the rate processes that determine the population of the electronic states. The description of these processes can be done only in an approximate way because of uncertainties concerning the rate coefficients, but it has been considered in some detail by BenDaniel and Tamor ${ }^{2}$ for conditions similar to those encountered here. When radiation processes are not important compared to the influence of electronic collisional processes in fixing the population of the electronic states, then the equilibrium approximation is valid. Judging from their results, the assumption of equilibrium appears to be valid for the experimental conditions. 
If the two-temperature model is used, the calculation of electron density and electrical conductivity given previously can be used, with the exception that the temperature that appears in these expressions must be the electron temperature. However, the electron temperature is now a variable and must be determined separately.

\section{Electron temperature}

For the quasi-steady state, electron temperature can be determined by considering the equation for conservation of energy for the system composed of the electron gas and the excited states. ${ }^{1}, 11$ The energy balance is given by equating the power supplied by the external field $J E$ to the losses. Two loss mechanisms are considered here; the most important is the elastic loss to atomic species. However, radiation losses must also be considered when $J E$ is small.

Elastic energy losses: The energy loss by electrons in elastic encounters with the atomic species can be expressed in terms of the diffusion cross sections occurring in the conductivity expressions. The treatment used here follows the development given by Petschek and Bryon. ${ }^{12}$ They assume that the atomic species have an isotropic distribution and that $m_{e} / m_{a} \ll 1$. If, in addition, we assume that the first term in the expansion for the electron distribution function is Maxwellian and note that for the potassium-argon system the masses of the atomic species are approximately equal, the rate of energy loss per unit volume from the electron component can be expressed as

$$
\dot{\Omega}=\frac{8}{3}\left(m_{e} / m_{a}\right)\left(\epsilon_{e}-\epsilon_{a}\right) n_{e} \Sigma_{m} \bar{\nu}_{m}
$$

where

$$
\bar{\nu} \equiv n_{i} \bar{c} \bar{Q}_{i}=n_{i} \bar{c} \int_{0}^{\infty} \frac{Q_{i}\{\xi\} \xi^{2} e^{-\xi} d \xi}{2}
$$

In terms of the conductivity, Eq. (7) may be written as

$\dot{\Omega}=\frac{m_{e}}{m_{a}}\left(\epsilon_{e}-\epsilon_{a}\right) n_{e}\left[\left(\frac{e^{2} n_{e}}{m_{e}}\right)\left(\frac{64}{3 \pi} \frac{\gamma_{E}}{\sigma_{s}}+\frac{64}{9 \pi} \frac{\sum_{n} n_{n} \bar{Q}_{n}}{\sum_{{ }_{n} n_{n} Q_{n}}} \cdot \frac{1}{\sigma_{c}}\right)\right]$

Steady-state radiation losses: The effects of inelastic collisions as an energy loss mechanism from the steady-state system of free and bound electrons are included in an approximate way by estimating radiation losses from the plasma due to bound-bound transitions. For the conditions of electron temperature and density of interest here, continuum radiation due to free-free and free-bound transitions is negligible compared to the bound-bound radiation losses. Thus, in estimating inelastic losses, the three-body electronic recombination process is assumed to be dominant over the two-body radiative process. In addition, the effects of three-body recombination with a heavy third body are ignored.

Several assumptions were made to estimate steady-state radiation losses. It was assumed that 1 ) the population levels of the excited states of potassium were in thermal equilibrium at the electron temperature; 2) spatial uniformity of absorbers and electron temperature existed throughout the plasma; 3 ) the gas volume was a cylinder of 1.91-cm length and of a diameter of $1.27 \mathrm{~cm}$; and 4) an equivalent mean beam length could be used in the conventional manner proposed by Hottel ${ }^{13}$ and extended by Olfe. ${ }^{14}$

The spectral line widths for this plasma are primarily fixed by Doppler broadening and dispersion broadening due to Lorentz and quadratic Stark effects. Broadening due to quadratic Stark effects was calculated by the approximate impact theory formula given by Margenau. ${ }^{15}$ Line widths due to Doppler and Lorentz broadening were computed by conventional formulas. Data given by Hinnov and $\mathrm{Kohn}^{16}$ and Mitchell and Zemansky ${ }^{17}$ were used to estimate optical cross sections for the Lorentz broadening of potassium lines

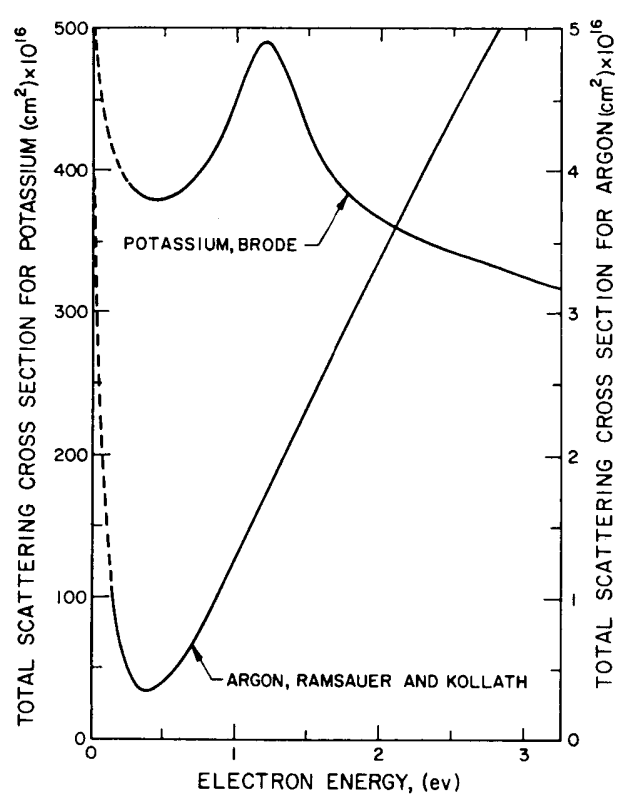

Fig. 3 Elastic collision cross sections.

by argon atoms. The effective half-width for dispersion effects was assumed to be the sum of the Lorentz and Stark half-widths. Transition probabilities were taken from many sources. ${ }^{18}$ Experimental data exist for the lines of primary importance and agree quite well with theoretical values found by the method of Bates and Damgaard. ${ }^{19}$

The method of calculation of line radiancies utilized the well-known "curves of growth" valid for the case of combined Doppler and dispersion broadening effects as outlined by Penner. ${ }^{20}$ Calculations of total radiancies were carried out for about 75 lines due to $s-p, p-d$, and $d-f$ transitions and for $2400 \leq T_{e} \leq 3500^{\circ} \mathrm{K}$ and $0.002 \leq n_{K} / n_{A} \leq 0.008$.

Energy balance: Combination of the results of the last paragraphs gives as the energy equation for the steady state:

$$
J E=J^{2} / \sigma=\frac{8}{3}\left(m_{e} / m_{a}\right)\left(\epsilon_{e}-\epsilon_{a}\right) n_{e} \Sigma_{m} \bar{\nu}_{m}+\dot{\leftrightarrow}_{s}
$$

where the radiation term is represented by $\dot{\mathfrak{R}}_{s}$.

The transient case is much more complex because the inelastic energy-loss terms depend in detail on the rate coefficients of the various energy transfer processes. A crude picture can be obtained by ignoring these terms completely. For this case, the energy of the electron gas is just $n_{e}\left(\epsilon_{e}+V_{i}\right)$, and the transient energy equation is given by

$(d / d t)\left[n_{e}\left(\epsilon_{e}+V_{i}\right)\right]=J E-\frac{8}{3}\left(m_{e} / m_{a}\right)\left(\epsilon_{e}-\epsilon_{a}\right) n_{e} \Sigma_{m} \bar{\nu}_{m}$

\section{Steady-State Calculations}

If we consider the quasi-steady condition described by Eq. (10), we can determine the electron temperature when the composition and temperature of the atomic species is specified. In practice, it is more convenient to pick an electron temperature and solve for $n_{e}$ from Eq. (6), $\sigma$ from Eq. (5), determine $\dot{\mathscr{Q}}_{a}$ as just described, and finally solve for $J$ and $E$ from Eq. (10). In all calculations of interest here, the quasi-steady state can be assumed to have been established in times short compared with the time in which appreciable changes have occurred in $T_{a}$. Hence, to a very good approximation, $T_{a}$ is the initial temperature of the atomic species.

The integral expressions for electrical conductivity and elastic collision losses due to close encounters were evaluated numerically using the total scattering cross-section values of Ramsauer and Kollath ${ }^{21}$ for argon and those of Brode $^{22}$ for potassium. The cross sections used are shown as a function of energy in Fig. 3. Unfortunately, these data are the total scattering cross section, whereas the diffu- 


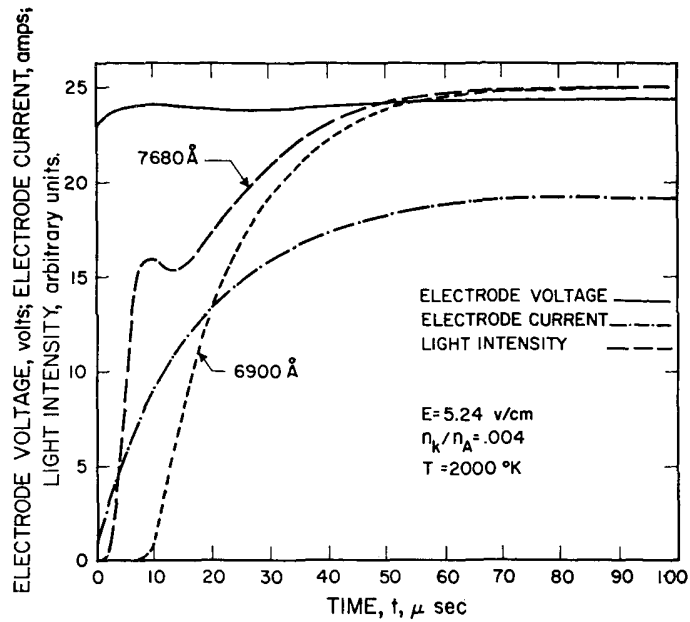

Fig. 4 A typical set of data.

sion cross section is required. However, in the range of electron energies of interest here, there is no pronounced forward or backward scattering, and there is probably less than a $10 \%$ difference between the two cross sections. ${ }^{23}$

Values of the conductivity and other parameters entering its determination are given in Table 1 for a range of electron temperatures and a potassium concentration of 0.004 . Several results are of interest. First, the close-encounter conductivity dominates at low temperatures and the Spitzer term at the high temperatures. Second, radiation loss is small compared to elastic-collision loss when the current density is above $4 \mathrm{amp} / \mathrm{cm}^{2}\left(T_{e} \approx 2800^{\circ} \mathrm{K}\right)$. For current densities below $1 \mathrm{amp} / \mathrm{cm}^{2}$, the radiation loss is dominant; and, for much smaller currents, the assumptions made concerning the two-temperature model probably fail. Third, for the conditions studied here, the calculated values for close-encounter conductivity could be closely approximated by use of energy-independent cross sections of magnitude $Q_{A}=0.70 \times 10^{-16} \mathrm{~cm}^{2}$ and $Q_{K}=400 \times 10^{-16} \mathrm{~cm}^{2}$. However, the averaged cross section that appears in the elastic loss term is temperature dependent.

\section{Transient}

The response of a plasma to a varying electric field can be analyzed by use of Eqs. (5), (11), and an expression for the net rate of production of electrons. Unfortunately, neither the detailed reaction mechanism nor the various cross sections for the reaction steps are sufficiently well known, and, consequently, not even approximate numerical solutions can be carried out. However, some information concerning the early stages of the response of a plasma to a step function

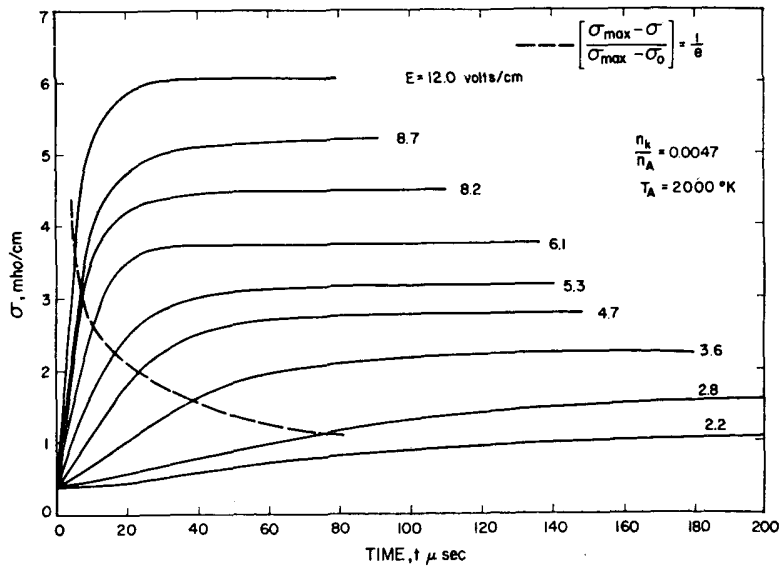

Fig. 5 Variation of conductivity with time. application of a field of strength $E$ can be obtained when inelastic losses are neglected. Equation (11) can be rearranged to give

$\sigma / n_{e} E^{2}=\left(V_{i}+\epsilon_{e}\right)\left(\dot{n}_{e} / n_{e}\right)+\dot{\epsilon}_{e}+\frac{8}{3}\left(m_{e} / m_{a}\right)\left(\epsilon_{e}-\epsilon_{a}\right) \Sigma_{m} \bar{\nu}_{m}$

and this equation can be analyzed to obtain several time constants.

First, assume that no energy goes into ionization. Then the first term on the right-hand side of Eq. (12) can be ignored, and the simplified equation can be integrated. The relaxation time for the establishment of the new electron temperature is given by an average value of $\left[\frac{8}{3}\left(m_{e} / m_{a}\right) \Sigma_{m} \bar{\nu}_{m}\right]^{-1}$ and is less than $3 \mu$ sec for these experiments.

Similarly, assume that all of the available energy goes into the ionization process. The second and third terms on the right-hand side of (12) are neglected, and the resulting integration indicates that the time required for the electron density to reach the steady-state value, fixed for the field $E$, is proportional to an average value of $\left(n_{c} V_{i} / \sigma E^{2}\right)$. Calculating this average value is complicated by the fact that $\sigma / n_{e}$ is a strong function of $n_{e}$ because of the ion-electron interaction term. For fields of the order of 3 to $10 \mathrm{v} / \mathrm{cm}$, numerical integration indicated that the relaxation time was in the range of 6 to 10 times the initial value of $\left(n_{e 0} V_{i} / \sigma_{0} E^{2}\right)$. For smaller fields, the multiplication factor is smaller, and, for a field of about $2.5 \mathrm{v} / \mathrm{cm}$, the factor is about 5 . For a field of $5 \mathrm{v} / \mathrm{cm}$, the time required is about $20 \mu \mathrm{sec}$.

Finally, assume that the electron density is constant and the neutral gas absorbs the power. The energy balance leads to the result that for a $10 \%$ change in neutral particle energy, a time of about $300 \mu$ sec is required when the field is $5 \mathrm{v} / \mathrm{cm}$.

These three calculations suggest the following picture of the transient process. During the first few microseconds, the electrons absorb an appreciable fraction of the power and reach an elevated temperature. Later, the ionization process is dominant and becomes the principal sink for energy. The ionization process remains the chief energy sink for most of the transient period, and, during this time, the ionization rate is fixed by the power input of the system. At the end of the transient, recombination processes dominate, and the rate of change of electron temperature and density becomes small. After this initial transient period, the temperature level of the whole system slowly increases.

\section{Experimental Results}

Measurements were made during the transient period immediately following the application of the voltage step function and also during the quasi-steady-state period after the transient had died out. The time required to reach this steady state was so short that phenomena associated with the gas flow or neutral heating could be ignored.

A typical set of observations are shown in Fig. 4. Here, current, voltage, and the intensity of the potassium resonance

Table 1 Variation of parameters with electron temperature $^{a}$

\begin{tabular}{llllccc}
\hline \hline$T_{e}$ & \multicolumn{1}{c}{${ }^{\circ} \mathrm{K}$} & 2400 & 2600 & 3000 & 3200 & 3500 \\
\hline$n_{e}$ & $10^{14} / \mathrm{cm}^{3}$ & 0.57 & 1.34 & 5.35 & 9.35 & 19.4 \\
$\sigma_{c}$ & $\mathrm{mho} / \mathrm{cm}$ & 0.526 & 1.22 & 4.60 & 7.81 & 16.1 \\
$\sigma_{s}$ & $\mathrm{mho} / \mathrm{cm}$ & 3.66 & 4.40 & 6.11 & 7.01 & 8.53 \\
$\sigma$ & $\mathrm{mho} / \mathrm{cm}$ & 0.46 & 0.96 & 2.6 & 3.7 & 5.6 \\
$\dot{\Omega}$ & $\mathrm{w} / \mathrm{cm}^{3}$ & 0.58 & 2.66 & 27.8 & 76.2 & 286 \\
$\dot{\leftrightarrow}_{s}$ & $\mathrm{w} / \mathrm{cm}^{3}$ & 1.53 & 2.80 & 8.40 & 13.5 & 25.8 \\
$J$ & $\mathrm{amp} / \mathrm{cm}^{2}$ & 0.98 & 2.29 & 9.74 & 18.2 & 41.1 \\
$E$ & $\mathrm{v} / \mathrm{cm}$ & 2.14 & 2.40 & 3.72 & 4.93 & 7.48 \\
\hline
\end{tabular}

${ }^{a} T_{a}=2000^{\circ} \mathrm{K}, n_{K} / n_{A}=0.004$, pressure $=1 \mathrm{~atm}$. 


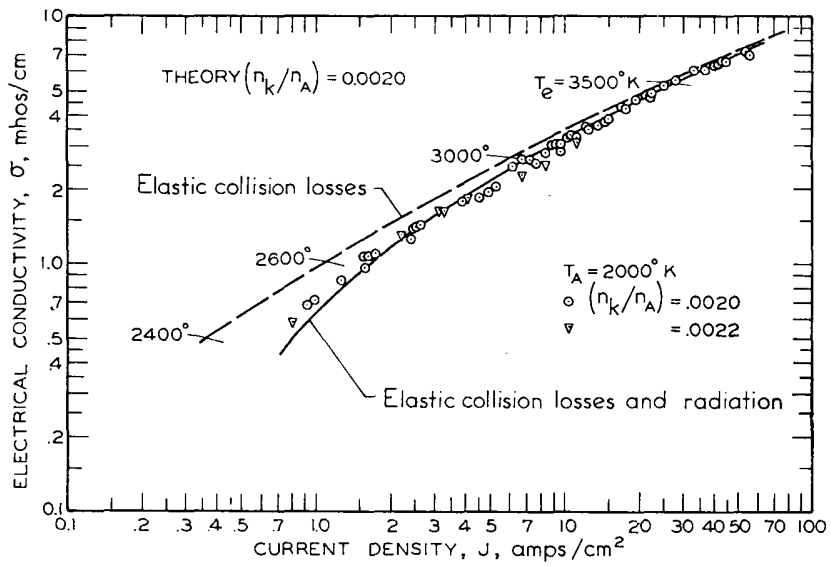

Fig. 6 Dependence of steady-state conductivity on current density.

lines at $7680 \AA$ and $6900 \AA$ are shown as a function of time. The voltage pulse is applied at zero time and remains approximately constant for the duration of the test. The current and spectral-line intensity rise sharply for small times and flatten off more gradually as the steady state is approached. The steady-state condition is reached after about $70 \mu \mathrm{sec}$, and the current and the line intensities reach a steady value at roughly the same time. Measured values of current and light intensity and inferred values of conductivity were found to be strong functions of the voltage gradient. Typical examples of the dependence of conductivity on time and voltage gradient are shown in Fig. 5. As the voltage gradient is increased, the steady-state conductivity values increase and the relaxation time decreases.

From data such as that presented in Figs. 4 and 5, it was possible to obtain steady-state values of the conductivity as a function of the voltage gradient or current density and to obtain values of the relaxation time for the conductivity change in response to a sudden change in field strength. In addition, some information concerning the mechanism of the ionization process was obtained from transient current measurements and preliminary light intensity data. In the following paragraphs, the steady-state results are discussed first.

\section{Steady-State Results}

\section{Conductivity}

The electric conductivity has been determined as a function of the applied field strength and the potassium concentration for a plasma with a temperature of $2000^{\circ} \pm 100^{\circ} \mathrm{K}$ and

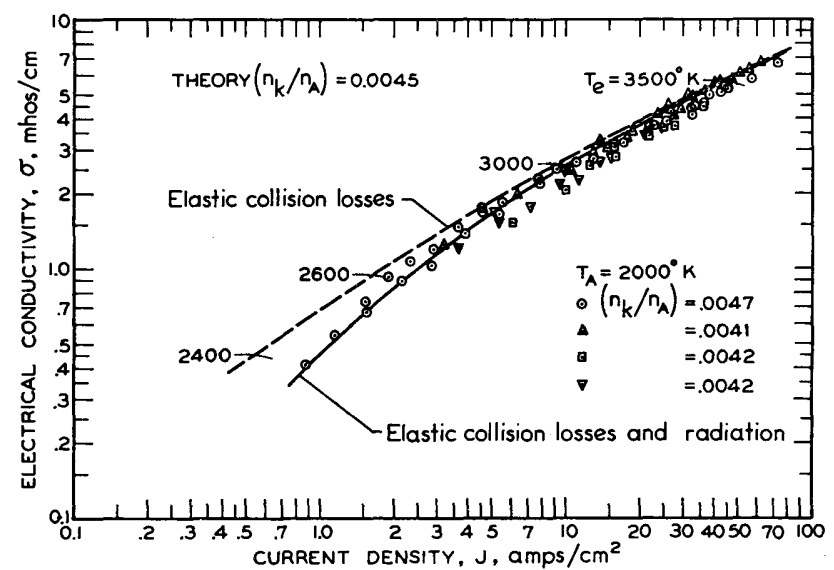

Fig. 7 Dependence of steady-state conductivity on current density.

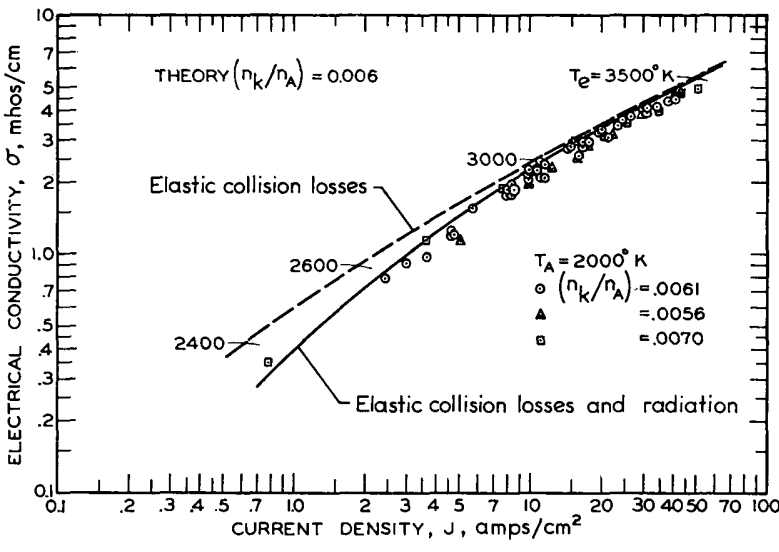

Fig. 8 Dependence of steady-state conductivity on current density.

a pressure of 1 atm. These values were obtained from steady-state results similar to those shown in Figs. 4 and 5 . The measurements were made over a 6 -month period and with a variety of changes in test-section geometry. About 200 values were obtained with good reproducibility.

The conductivity data are shown in Figs. 6 to 9 for four values of potassium concentration and are plotted as a function of current density. The current density covers the range from 0.8 to about $90 \mathrm{amp} / \mathrm{cm}^{2}$ with a variation of conductivity between 0.5 and $8 \mathrm{mho} / \mathrm{cm}$. Values of conductivity calculated by the two-temperature theory discussed earlier are presented as a curve on each figure. The data and theory agree very well over the whole range, and it is evident that the radiation correction is very important at the low current densities.

The agreement between experimental and theoretical values is much better than could be expected considering the experimental uncertainties and simplifying assumptions made in the theory. Some of the experimental uncertainties are due to the possible effects of radial temperature gradients, impurities in the argon, and errors in potassium concentration and plasma temperature. The theoretical values may be incorrect because of the many simplifying assumptions made in the theory and because of errors in cross-section values. Given these possible errors, one can only conclude that the conductivity values are not very sensitive to the inaccuracies listed here. This is largely true because $\sigma$ is almost proportional to $J$. A more precise check on the proposed two-temperature model must depend on electron density and electron temperature measurements.

Some effort has been made to measure the electron temperatures directly by use of the spectral-line intensity data and the sodium-line reversal technique. The data give

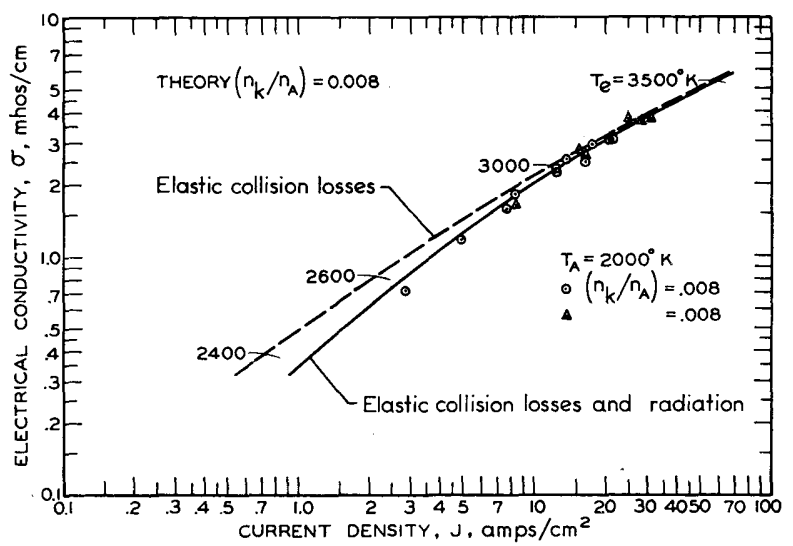

Fig. 9 Dependence of steady-state conductivity on current density. 
values that are about $10 \%$ below the theoretical values, e.g., $2870^{\circ} \mathrm{K}$ measured and $3200^{\circ} \mathrm{K}$ expected at $J=20$ $\mathrm{amp} / \mathrm{cm}^{2}$ and $3000^{\circ} \mathrm{K}$ measured and $3320^{\circ} \mathrm{K}$ expected at $J=30 \mathrm{amp} / \mathrm{cm}^{2}$. It is not obvious yet whether this discrepancy is due to experimental technique or some error in theory.

\section{Transient Data}

\section{Relaxation times}

The information most directly obtained from the transient measurements is the determination of the relaxation times for the conductivity of a plasma in response to the application of a step function in the electric field. This information is obtained from data plots similar to Fig. 5. It is clear from this figure that, as the field strength is increased, the steadystate values of conductivity increase and the time required to reach these values decreases.

In analyzing the data, it is convenient to give the results as a function of a characteristic time for the process. The discussion of the energy equation (12) under the "TwoTemperature Model" suggests that the group $\left(n_{e 0} V_{i} / \sigma_{0} E^{2}\right)$ forms the appropriate characteristic time.

The relaxation time $\tau_{r}$ was taken as the time required for the change in conductivity, $\left(\sigma-\sigma_{0}\right)$, to reach $(1-1 / e)$ of its final value $\left(\sigma_{\max }-\sigma_{0}\right)$. Values of $\tau_{r}$ are given in Fig. 10 for a range of values of potassium concentration and as a function of the characteristic time, $\left(n_{e 0} V_{i} / \sigma_{0} E^{2}\right) \equiv \tau_{c}$. The data lie roughly along a straight line through the origin with a slope of about 8 . For the experimental conditions used here, a field of $4 \mathrm{v} / \mathrm{cm}$ and $n_{K} / n_{A}=0.0042$ gives a characteristic time of about $4 \mu \mathrm{sec}$ and a relaxation time of about $30 \mu$ sec. Higher fields correspond to much shorter times, and, for strengths as high as $10 \mathrm{v} / \mathrm{cm}$, the relaxation time is less than $10 \mu \mathrm{sec}$. It is evident, then, that relaxation times for field strengths of interest will be in the range of tens of microseconds or less.

For a given field strength, the characteristic time changes by about a factor of 3 over the range of potassium concentrations covered by the experiments. The good correlation of the data of Fig. 10 suggests that the dependence on potassium concentration is correctly accounted for by use of the characteristic times as the variable.

Note that, for large fields, measured values of relaxation times are almost identical with the theoretical values found in the preceding section when the assumption was made that all available power went into ionization. Both depend linearly on $\tau_{c}$, and both are about 8 times larger. Thus, it appears that the ionization rate, and hence the rate of change of conductivity, is given by the rate of power input for fields

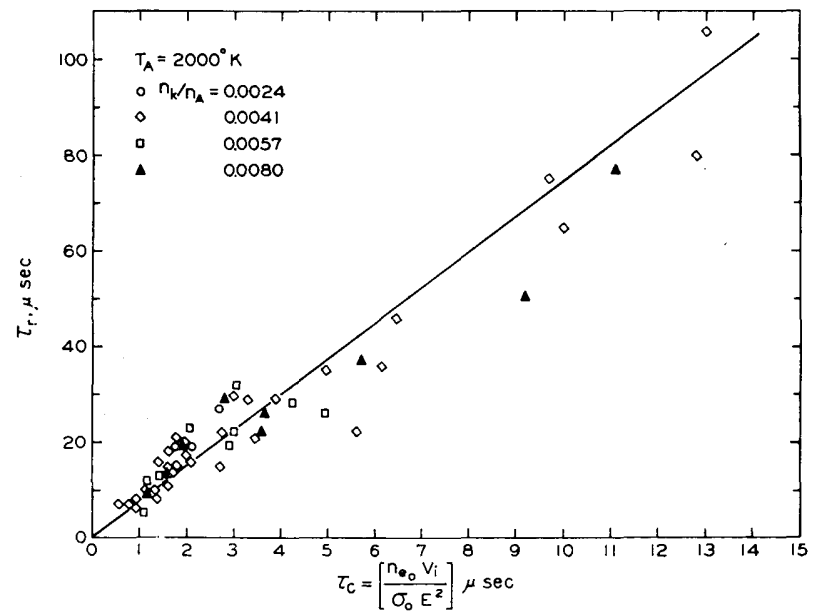

Fig. 10 Variation of relaxation time with characteristic time. in the range $3.5 \leq E \leq 10 \mathrm{v} / \mathrm{cm}$. For field strengths less than $3.5 \mathrm{v} / \mathrm{cm}$, the experimentally-determined relaxation times are larger than those predicted from the assumption that all the power goes into ionization, and it is evident that, for these low fields, elastic energy and radiation losses are important sinks for power during the ionization period.

The small values found for the relaxation times imply that no transient conductivity problems will be encountered at the entrance of an electric generator using a seeded plasma. For example, with a field strength of $3 \mathrm{v} / \mathrm{cm}$, the relaxation length for a flow with a Mach one inlet speed, $n_{K} / n_{A}=$ 0.0028 , and $T_{a}=2000^{\circ} \mathrm{K}$ is about $4 \mathrm{~cm}$. Normal fringing of the electric field should obscure the presence of such a relaxation length and make its effects negligible.

\section{Relaxation mechanism}

The relaxation times for the conductivity observed in this experiment show that steady ionization levels are reached in times too short to be explained only on the basis of direct ionization of unexcited atoms. Using a linear approximation to the low-energy portion of the cross section vs energy curves for ionization from the ground state and the cross section estimated from data given by von Engel, ${ }^{24}$ one would calculate that the relaxation time would be greater than $4 \times$ $10^{-4}$ sec for $T_{e f}=3500^{\circ} \mathrm{K}$. Experimentally, we observe conductivity relaxation times that are a factor of 40 times smaller than this. Thus, it appears that multistep ionization processes are important in the potassium-argon system under our experimental conditions.

Some additional information concerning the relaxation mechanism early in the pulse was obtained from the values of light intensity measured for the two spectral regions and from the examination of the initial rate of change of conductivities. The spectral data (Fig. 4) indicate that the population of the first excited state, and hence by inference, the electron temperature, rises much more rapidly than the conductivity during the first few microseconds. In contrast, higher populated states rise much more slowly.

Combination of measured values of the initial rate of change of conductivity and Eq. (12) make it possible to determine the important terms in the energy balance early in the pulse. The analysis shows that, for $E>3.5 \mathrm{v} / \mathrm{cm}$, the dominant term is the ionization process and, for $E<3.0 \mathrm{v} /$ cm, either $\dot{\mathscr{R}}_{8}$ or $\dot{\Omega}$ was dominant.

These data, discussed much more fully in Ref. 25 , indicate that the general features of the ionization process are similar to those suggested in the theoretical discussion. That is, the electron temperature reaches an elevated value very early in the process, and the chief energy sink thereafter is the ionization process. They also indicate that, when the field strength becomes sufficiently small, this general picture breaks down, and heating of the neutral gas or energy loss by radiation appears to become dominant.

\section{Summary}

The electrical conductivity of a plasma, made up of argon seeded with potassium vapor, has been determined experimentally for a wide range of current density and potassium concentration and for a temperature of $2000^{\circ} \mathrm{K}$. The experimental values agree very well with values calculated from the two-temperature model. Electron temperature measurements were about $10 \%$ below values estimated from conductivity data and theory. These results confirm the usefulness of the two-temperature model for calculation of the nonequilibrium conductivity, but more precise measurements of electron density and temperature are required to evaluate properly the general validity of the model.

Values of the relaxation time for the conductivity in response to a step function change in electric field have been determined for the range of conditions just described. The 
values were found to be proportional to $\left(n_{e 0} / \sigma_{0} E^{2}\right)$ and were in the range of a few tens of microseconds for field strengths greater than $3 \mathrm{v} / \mathrm{cm}$. When the field strength was above 3 $\mathrm{v} / \mathrm{cm}$, the experimental values of relaxation times were similar in magnitude and dependence on $\left(n_{e 0} / \sigma_{0} E^{2}\right)$ to values estimated on the basis of the assumption that all the power went directly into the ionization process. The short relaxation times observed suggest that ionization occurs by a multistep process. Observations made during the transient period indicate that the electron temperature rises to an appreciable fraction of the steady-state value during the first $5 \mu \mathrm{sec}$ of the voltage pulse. This result is in good agreement with the short times observed for relaxation.

\section{References}

${ }^{1}$ Kerrebrock, J. L., "Conduction in gases with elevated electron temperatures," Engineering Aspects of Magnetohydrodynamics (Columbia University Press, New York, 1962), pp. 327-346; also Kerrebrock, J. L., "Non-equilibrium effects on conductivity and electrode heat transfer in ionized gases," Guggenheim Jet Propulsion Center, California Institute of Technology, TN 4 (November 1960).

${ }^{2}$ BenDaniel, D. J. and Tamor, S., "Non-equilibrium ionization in magnetohydrodynamic generators," General Electric Tech. Information Series, 62-RL-(2922E) (January 1962).

${ }^{3}$ Robben, F., "Nonequilibrium ionization in a magnetohydrodynamic generator," Phys. Fluids 5, 1308 (1962).

${ }^{4}$ Pinchak, A. C., "Experimental investigation of gas phase and surface phenomena in a seeded plasma," Ph.D. Thesis, California Institute of Technology, Pasadena, Calif. (June 1963).

${ }^{5}$ Pinchak, A. C. and Zukoski, E. E., "Gas phase and surface phenomena observed with a seeded plasma," Proceedings of the 4th Symposium on the Engineering Aspects of Magnetohydrodynamics (University of California Press, Berkeley, Calif., 1963), pp. 51-57.

${ }^{6}$ Chapman, S. and Cowling, T. G., The Mathematical Theory of Non-Uniform Gases (Cambridge University Press, London, 1961), Chap. 7.

${ }^{7}$ Allis, W. P., "Motions of ions and electrons," Handbuch der Physik (Springer-Verlag, Berlin, 1956), Vol. XXI, pp. 383-444.

${ }^{8}$ Lin, S. C., Resler, E. L., and Kantrowitz, A., "Electrical conductivity of highly ionized argon produced by shock waves," J. Appl. Phys. 26, 95-109 (1955)

${ }^{9}$ Spitzer, L., Jr., Physics of Fully Ionized Gases (Interscience Publishers, New York, 1956). Chap. 5.
${ }^{10}$ Frost, L. S., "Conductivity of seeded atmospheric pressure plasmas," J. Appl. Phys. 32, 2029-2036 (1961).

${ }^{11}$ Massey, H. S. W. and Burhop, E. H. S., Electronic and Ionic Impact Phenomena (Oxford University Press, London, 1956), p. 12

${ }^{12}$ Petschek, H. and Bryon, S., "Approach to equilibrium ionization behind strong shock waves in argon," Ann. Phys. 1, 270-315 (1957).

${ }^{13}$ Hottel, H. C., "Radiant-heat transmission," Heat Transmission, edited by W. H. MacAdams (McGraw-Hill Book Co. Inc., New York, 1954), Chap. 4.

${ }_{14}$ Olfe, D. B., "Mean beam length calculations for radiation from nontransparent gases," J. Quant. Spectr. Radiative Transfer 1, 169-176 (1961).

${ }^{15}$ Margenau, H., "Formulas for estimating widths of spectral lines emitted from plasmas and their limits of validity," Proceedings of the 4th International Conference on Ionization Phenomeno in Gases (North-Holland Publishing Co., Amsterdam, 1960), Vol. 2, p. 799 .

${ }^{16}$ Hinnov, E. and Kohn, H., "Optical cross sections from intensity-density measurements," J. Opt. Soc. Am. 47, 156-162 (February 1957)

${ }_{17}$ Mitchell, A. C. G. and Zemansky, M. W., Resonance Radiation and Excited Atoms (Cambridge University Press, London, 1934), p. 179.

${ }^{18}$ Glennon, B. M. and Wiese, W. L., "Bibliography on atomic transition probabilities," Natl. Bur. Std. (U. S.) Monograph 50 (August 1962).

${ }^{19}$ Bates, D. R. and Damgaard, A., "The calculation of the absolute strengths of spectral lines," Phil. Trans. Roy. Soc. London A242, 101-122 (1949).

${ }^{20}$ Penner, S. S., Quantitative Spectroscopy and Gas Emissivities (Addison-Wesley Publishing Co., Inc., Reading, Mass., 1959), Chap. 4.

${ }^{21}$ Ramsauer, V C. and Kollath, R., "Winkelverteilung bei der Streuung Langsamer Electronen an Gasmolekülen," Ann. Phys. 12, 529-561 (1932).

${ }_{22}$ Brode, R. B., "Absorption coefficient for slow electrons in alkali metal vapors," Phys. Rev. 34, 673-678 (1929).

${ }^{23}$ Brown, S. C., Basic Data of Plasma Physics (MIT Press, Cambridge, Mass., and John Wiley and Sons, Inc., New York, 1959), p. 31 .

${ }^{24}$ von Engel, A., Ionized Gases (Clarendon Press, Oxford, England, 1955), p. 52.

${ }^{25}$ Zukoski, E., Cool, T., and Gibson, E., "Non-equilibrium conductivity in a seeded plasma," Guggenheim Jet Propulsion Center Rept., California Institute of Technology, Air Force Office of Scientific Research, AFOSR 64-0848 (May 1964). 